\title{
Multiplicity of scision neutrons from density functional scission dynamics
}

\author{
Nicolae Carjan ${ }^{1,2, *}$, Ionel Stetcu ${ }^{3}$, Margarit Rizea ${ }^{2}$, and Aurel Bulgac ${ }^{4}$ \\ ${ }^{1}$ Joint Institute of Nuclear Research, Dubna, Russia \\ 2"Horia Hulubei" National Institute for Physics and Nuclear Engineering, Bucharest, Romania \\ ${ }^{3}$ Theoretical Division, Los Alamos National Laboratory, Los Alamos, NM 87545, USA \\ ${ }^{4}$ Department of Physics, University of Washington, Seattle, Washington 98195, USA
}

\begin{abstract}
The time evolution of the nuclear density of the fissioning system ${ }^{240} \mathrm{Pu}$ during the scission process is obtained from the time-dependent superfluid local-density approximation (TDSLDA) to the density functional theory. A nuclear energy density functional based on the Skyrme force Skm* is used. The duration of the scission process $\Delta t$ as well as the neck radius $\left(r_{\min }\right)$ of the 'just-before scission' configuration and the minimum separation $\left(d_{\min }\right)$ of the inner surfaces of the fragments in the 'immediately-after scission' configuration were extracted in order to calculate the multiplicity of the scission neutrons $\left(v_{\mathrm{sc}}\right)$ using a phenomenological dynamical scission model (DSM). We find that $v_{\mathrm{sc}}=1.347$, i.e. half of the prompt fission neutrons measured in the reaction ${ }^{239} \mathrm{Pu}\left(n_{\mathrm{th}}, f\right)$ are released at scission. After scission, the fragments are left excited and with some extra deformation energy (mainly the heavy one). In this way we can account for the evaporation of the other half and for the emission of $\gamma$ rays.
\end{abstract}

\section{Introduction}

The most important feature of nuclear fission is that it is accompanied by emission of prompt fission neutrons (PFN). Two PFN sources have been discussed so far:

1) Neutrons evaporated from fully-accelerated excited fragments (EVN).

2) Neutrons dynamically released at scission (SN) due to the diabatic coupling between the neutron degree of freedom and the rapidly changing neutron-nucleus potential.

The relative intensities of these two components are unknown. It is very difficult to separate them experimentally since the gross features of PFN can be accounted for by both hypotheses.

While the 1st component depends on the excitation and extra deformation with which the fission fragments are born [1], the 2nd depends on the dynamical evolution of the system during the scission process [2]. Therefore a simple stationary approach suffices for EVN but a more complicated dynamical approach is needed for SN.

There are three parameters in the dynamical scission model: the nuclear shapes just before $\left(\alpha_{i}\right)$ and immediatelly after $\left(\alpha_{f}\right)$ scission and the duration $\Delta T$ of the transition between these two shapes. Since these quantities were unknown, in the past we have used educated guesses.

\footnotetext{
*e-mail: carjan@theory.nipne.ro
} 
At present reliable values for these quantities are available from the density functional theory (DFT) applicable to superfluid systems (usually named the superfluid local density approximation or SLDA), within a time-dependent extension (TD-SLDA) [3]. Among the many nuclear energy density functionals we choose the one based on the Skyrme force SkM* [4] that reproduces the fission barriers in ${ }^{240} \mathrm{Pu}$. We extract these three quantities and calculate the multiplicity of the neutrons released at scission and emitted immediately after (i.e., during the acceleration of the fragments).

Finally we also estimate the energy available to emit prompt $\gamma$ rays and evaporate neutrons: $E_{\mathrm{sc}}^{*}+\Delta E_{\mathrm{def}}$

\section{Dynamical scission model (DSM)}

To simulate the evolution of the neutron wave functions from $\alpha_{i}$ to $\alpha_{f}$ lasting $\Delta T$, the twodimensional time-dependent Schrödinger equation (TDSE2D) with a time-dependent potential (TDP) is solved. The distribution of final wave packets $\hat{\Psi}^{i}(\Delta T)$ (that correspond at $t=0$ to the eigenstates $\hat{\Psi}^{i}$ of $\alpha_{i}$ ) over the eigenstates $\hat{\Psi}^{f}$ of $\alpha_{f}$ :

$$
a_{i f}=\left\langle\hat{\Psi}^{i}(\Delta T) \mid \hat{\Psi}^{f}\right\rangle
$$

constitutes the core of DSM. All wave functions have an implicit dependence on the cylindrical coordinates $(\rho, z) . a_{i f}$ is $\neq 0$ only if $\left|\hat{\Psi}^{i}\right\rangle$ and $\left|\hat{\Psi}^{f}\right\rangle$ have the same projection $\Omega$ of the total angular momentum along the symmetry axis.

The rapid change of the potential in which the nucleons move produces, by means of a diabatic coupling, their excitation and eventual emission.

The $a_{i f}$ coefficients are used to calculate the scission neutron multiplicity $v_{\mathrm{sc}}$ in a simple and intuitive way. It is given by the sum of the probabilities $P_{\mathrm{em}}^{i}$ that a neutron occupying a given bound-state $\mathrm{i}$ is emitted:

$$
\begin{gathered}
P_{\mathrm{em}}^{i}=v_{i}^{2} \sum_{f}\left(1-\left|a_{i f}\right|^{2}\right) \\
v_{\mathrm{sc}}=2 \sum_{i} P_{\mathrm{em}}^{i}
\end{gathered}
$$

The sums are over the bound states of the initial and final configurations. $v_{i}^{2}$ is the occupation probability of the state $i$.

Similarly, the excitation energy in which the fragments are left immediately after scission is given by

$$
E_{\mathrm{sc}}^{*}=2 \times\left(\sum_{f} V_{f}^{2} e_{f}-\sum_{f} v_{f}^{2} e_{f}\right),
$$

where $V_{f}^{2}=\sum_{i} v_{i}^{2}\left|a_{i f}\right|^{2}$ is the probability that an eigenstate $\left|\hat{\Psi}^{f}\right\rangle$ of the final configuration $\alpha_{f}$ is occupied after the sudden transition and $e_{f}$ is its eigen-energy. The $\mathrm{i}$ and $\mathrm{f}$ sums are over the bound states of the initial and final nuclear configurations, respectively. The nuclear shapes involved are described by Cassini ovals [5] with two parameters: $\alpha$ (the overall deformation) and $\alpha_{1}$ that fixes the mass asymmetry.

\section{TD-SLDA}

In SLDA, the nuclear system is described by a density functional $\mathcal{E}(n(\vec{r}), v(\vec{r}), \vec{j}(\vec{r}), \ldots)$, where $n$ is the normal (number) density, $v$ is the anomalous density, $\vec{j}$ is the current density, etc. They are defined in terms of the $u_{k \uparrow(\downarrow)}$ and $v_{k \uparrow(\downarrow)}$ components of the quasiparticle wave functions 
(qpwfs). The dynamics of the nucleus is followed in real time by solving a system of timedependent Schrödinger-like equations

$$
\begin{gathered}
i \hbar \frac{\partial}{\partial t}\left(\begin{array}{c}
u_{k \uparrow} \\
u_{k \downarrow} \\
v_{k \uparrow} \\
v_{k \downarrow}
\end{array}\right)= \\
\left(\begin{array}{cccc}
h_{\uparrow \uparrow}-\mu & h_{\uparrow \downarrow} & 0 & \Delta \\
h_{\downarrow \uparrow} & h_{\downarrow \downarrow}-\mu & -\Delta & 0 \\
0 & -\Delta^{*} & -\left(h_{\uparrow \uparrow}^{*}-\mu\right) & -h_{\uparrow \downarrow}^{*} \\
\Delta^{*} & 0 & -h_{\downarrow \uparrow}^{*} & -\left(h_{\downarrow \downarrow}^{*}-\mu\right)
\end{array}\right)\left(\begin{array}{c}
u_{k \uparrow} \\
u_{k \downarrow} \\
v_{k \uparrow} \\
v_{k \downarrow}
\end{array}\right),
\end{gathered}
$$

with the dependence on the spacial coordinates and time not shown explicitly. The onebody Hamiltonian $h=\partial \mathcal{E} / \partial v^{*}$ and the pairing field $\Delta$ depend on the densi- ties and currents constructed from the qpwfs at the time $t$.

\subsection{TD-SLDA scission dynamics}

We calculate at each time the number density

$n(\vec{r}, t)=\sum_{k}\left(\left|v_{k \uparrow}(\vec{r}, t)\right|^{2}+\left|v_{k \downarrow}(\vec{r}, t)\right|^{2}\right)$ shown in Fig. 1 at several times before and after scission for ${ }^{240} \mathrm{Pu}$. This sequence of densities leads to a mass asymmetry of the separated fragments defined by $A_{L}=104\left(A_{H}=136\right)$ close to the most probable experimental mass division in the reaction ${ }^{239} \mathrm{Pu}\left(n_{\mathrm{th}}, f\right)$. The middle equidensity line is the half density $n_{0} / 2$ with $n_{0}=0.16$ $\mathrm{fm}^{-3}$. It is equivalent with the equipotential line $V_{0} / 2$ of the deformed Woods-Saxon potential used in DSM. $V_{0}$ is the potential depth. These equipotential lines are shown for comparison on the same figure. One can notice a good similarity between the nuclear shapes used in the two models.

\subsection{The limits of the scission process}

To determine when the scission process starts and when it ends we analyze the evolution of the moments of the density distribution, in particular we look for the sharp decrease of the total quadrupole and for the oscillation of the fragment octupoles (see Fig. 2).

\section{Parametrization of the scission process}

One can estimate that the rapid transition at scission starts at $\approx 800 \mathrm{fm} / \mathrm{c}$ and lasts untill $\approx 1200 \mathrm{fm} / \mathrm{c}$, its duration being $\Delta T=13.4 \times 10^{-22} \mathrm{sec}$. Going back to Fig. 1 , one can see that the corresponding configurations have $r_{\min }=2.13 \mathrm{fm}$ and $d_{\min }=11.48 \mathrm{fm}$ respectively. $r_{\min }$ and $d_{\min }$ are the neck radius and the separation of the inner surfaces of the fragments respectively.

This inference is also supported by the variation of the octupole moments $Q_{z z z}$ of the $\mathrm{L}$ and $\mathrm{H}$ fragments from Fig. 2. 

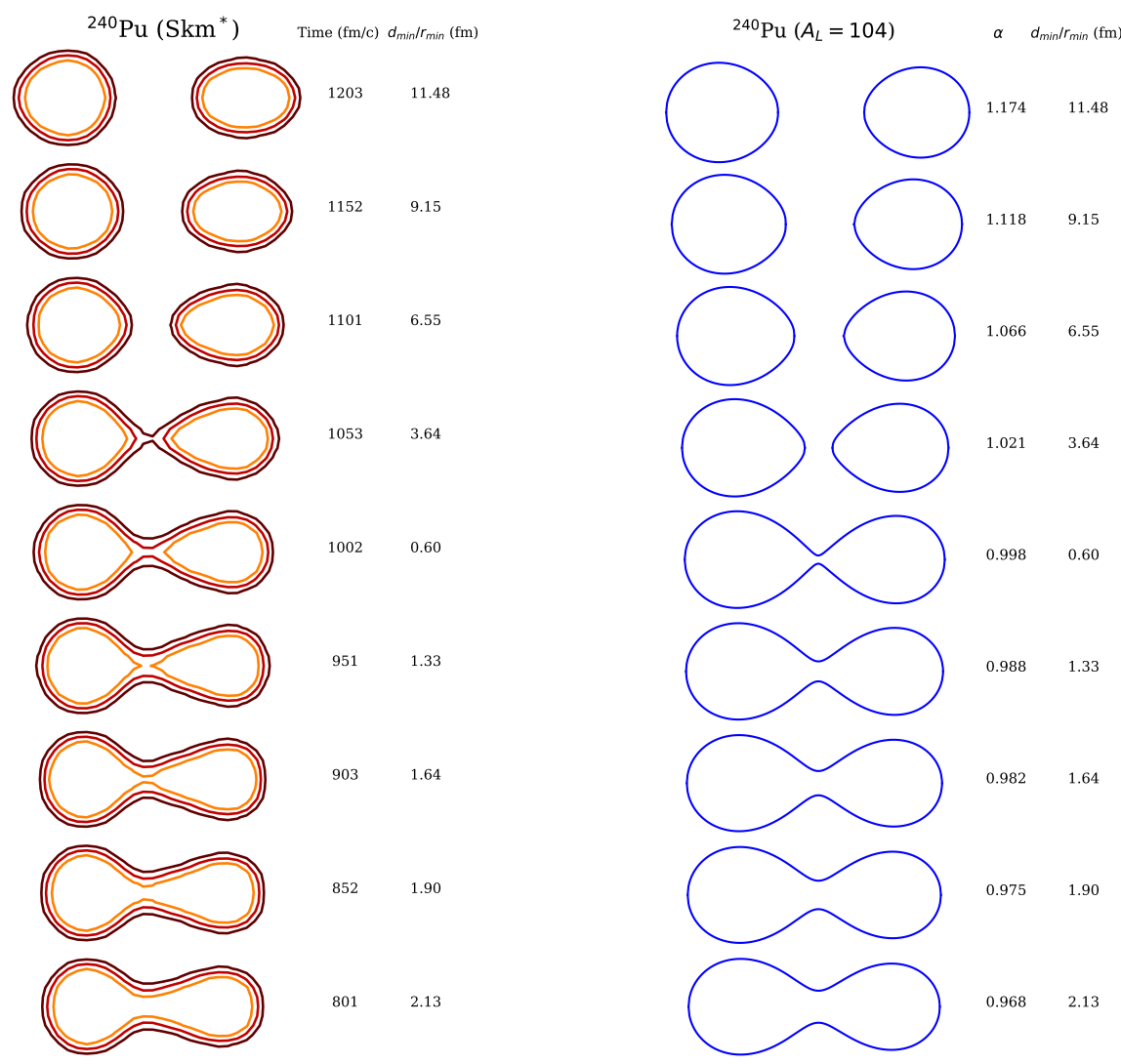

Figure 1. Time evolution of the density (left column) and of the potential (right column).

Table 1. Scission neutron multiplicity: ${ }^{240} P u, A_{L}=104,0.968>>1.174, \Delta T=13.4 \times 10^{-22} \mathrm{sec}$.

\begin{tabular}{|c|c|c|c|c|c|c|c|}
\hline$\Omega$ & $1 / 2$ & $3 / 2$ & $5 / 2$ & $7 / 2$ & $9 / 2$ & $11 / 2$ & $\Sigma(\Omega)$ \\
\hline$v_{\mathrm{sc}}$ & 0.833 & 0.465 & 0.037 & 0.005 & 0.006 & 0.001 & 1.347 \\
\hline
\end{tabular}

\subsection{SCN multiplicities}

The deformations of the Cassini ovals that have the same $r_{\min }$ and $d_{\min }$ values are found (Fig. 1) to be $\alpha_{i}=0.968$ and $\alpha_{f}=1.174$ respectively. We therefore calculate the evolution of all neutron wave functions from $\alpha_{i}$ to $\alpha_{f}$ keeping the mass asymmetry fixed at $A_{L}=104$ with the help of the parameter $\alpha_{1}$. The duration of the transition is $\Delta T=13.410^{-22} \mathrm{sec}$.

\subsection{SCN, EVN and $\gamma$ rays}

The resulting scission-neutron multiplicities are presented for each set of wave functions of given $\Omega$ in Table 1. $v_{\mathrm{sc}}$ decreases with $\Omega$, the contribution of $\Omega=1 / 2$ being more then $50 \%$. In the case of ${ }^{240} \mathrm{Pu}$ the multiplicity summed over all $\Omega$ values is found to be 1.347 , i.e., half of the prompt fission neutrons measured in the reaction ${ }^{239} \mathrm{Pu}\left(n_{\mathrm{th}}, f\right)$ are released at scission. 


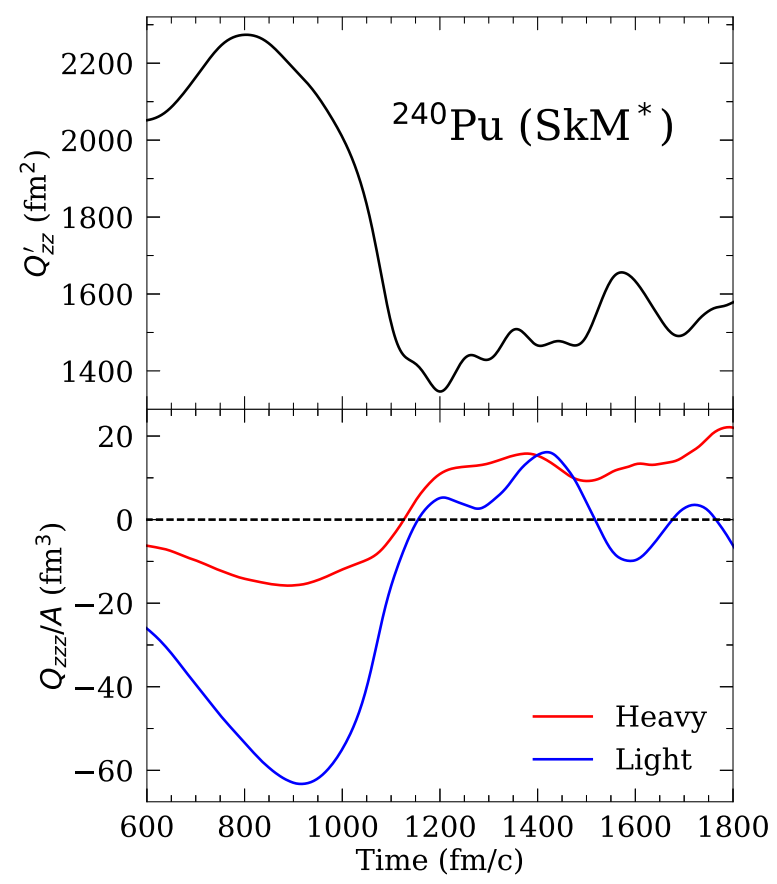

Figure 2. Evolution of the quadrupole moment (of the whole system) and of the octupole moment (of the fragments) from before to after scission.

Table 2. Deformation parameters of the primary fragments and their extra deformation energy:

$$
{ }^{240} \mathrm{Pu}, A_{L}=104 \text {. }
$$

\begin{tabular}{|c|c|c|c|c|}
\hline$Z$ & $A$ & $\beta_{2}$ & $\beta_{3}$ & $\Delta E_{\text {def }}$ \\
\hline 41 & 104 & 0.39 & 0.10 & $0.79 \mathrm{MeV}$ \\
\hline 53 & 136 & 0.10 & 0.10 & $2.27 \mathrm{MeV}$ \\
\hline
\end{tabular}

In addition of being promoted to positive energy states at the end of the scission process, the neutrons are also promoted to states between the Fermi and the "zero" level producing excitation in the primary fragments. Eq. (3) gives $E_{\mathrm{sc}}^{*}=12.59 \mathrm{MeV}$.

Finally we estimate the extra deformation energy $\Delta E_{\text {def }}$ of the primary fragments. From the figures with the time evolution of $Q_{z z}$ and $Q_{z z z}$ of each fragment, one can extract their deformations $\beta_{2}$ and $\beta_{3}$ (see Fig. 3) and from this we calculate their post-scission deformation energies [6]. The results are summarized in Table 2.

On one side we have the estimated total excitation energy

$$
E_{\mathrm{sc}}^{*}+\Delta E_{\mathrm{def}}=15.65 \mathrm{MeV}
$$

that will be used to emit prompt $\gamma$ rays and evaporate neutrons.

On the other side we have $\left\langle E_{\gamma}\right\rangle=6.70 \mathrm{MeV}$ measured [7] and 1.53 neutrons to evaporate. We use experimental masses to calculate neutron separation energies. $S_{n}=4.97 \mathrm{MeV}$ and $3.78 \mathrm{MeV}$ for the $\mathrm{L}$ and $\mathrm{H}$ fragments for the odd-odd $\mathrm{L}$ and $\mathrm{H}$ fragments from Table 2. 


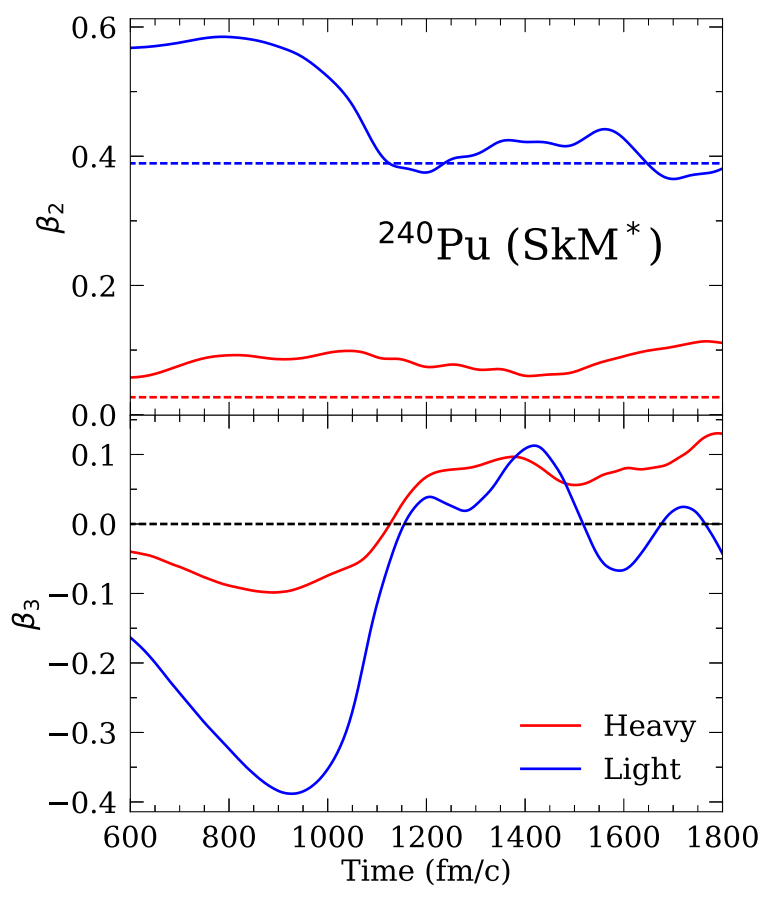

Figure 3. Evolution of $\beta_{2}$ and $\beta_{3}$ deformations of the nascent fragments.

For neighbouring even-even fragments $S_{n}=7.55 \mathrm{MeV}$ and 4.67 MeV. Using an average value for $S_{n}$, the evaporation of 1.53 neutrons requires $8.02 \mathrm{MeV}$. So the sum is $14.72 \mathrm{MeV}$.

In view if the uncertainties in the model one can consider the agreement between the total excitation energy calculated $(15.65 \mathrm{MeV})$ and the energy carried by evaporated neutrons and gammas (14.72 MeV) satisfactory.

\section{Discussion and conclusions}

- We give a first plausible answer to a long-standing question regarding the emission of prompt fission neutrons: is the percentage of neutrons emitted at scission and during acceleration significant? This answer is YES!

- We use DSM to calculate $v_{\mathrm{sc}}$ and $E_{\mathrm{sc}}^{*}$ In this phenomenological model the dynamics of the neck rupture determines how many unbound neutrons and how much excitation we have at the end.

- Implementing the scission dynamics given by TD-SLDA into DSM, we obtain $v_{\mathrm{sc}}=$ 1.347 (half of the PFN originate at scission) and $E_{\mathrm{sc}}^{*}=12.59 \mathrm{MeV}$.

- From the deformations of the primary fragments predicted by TD-SLDA, we calculated their extra deformation energies $\Delta E_{\text {def }}=3.06 \mathrm{MeV}$.

- The total energy balance is fulfilled to a good approximation.

We are grateful to Patrick Talou for his interest in this work and very useful discussions.

\section{References}

[1] D.G. Madland, J.R. Nix, Nucl. Sci. Eng. 81, 213 (1982) 
[2] N. Carjan, M. Rizea, Phys. Rev. C 82, 014617 (2010)

[3] A. Bulgac, P. Magierski, K.J. Roche, I. Stetcu, Phys. Rev. Lett. 116, 122504 (2016)

[4] J. Bartel, P. Quentin, M. Brack, C. Guet, H.-B. Håkansson, Nucl. Phys. A 386, 79-100 (1982)

[5] V.V. Pashkevich, Nucl. Phys. A 169, 275-293 (1971)

[6] P. Möller, A.J. Sierk, T. Ichikawa, H. Sagawa, Atomic Data and Nuclear Data Tables, 109-110, 1-204 (2016)

[7] H.H. Knitter, U. Brosa, C. Butz-Jorgensen, The Nuclear Fission Process (CRC Press, Boca Raton USA, 1991), p. 497 